\title{
CORREÇÃO DE RÁDIO CURVO POR TÉCNICA DE OSTECTOMIA EM CUNHA E OSTEOSSÍNTESE COM PLACA E PARAFUSOS: RELATO DE CASO
}

\author{
BRASIL, Fabrício B. J $\mathbf{J}^{1}$ \\ MENDES, Patrícia F. ${ }^{2}$ \\ SILVA, Letícia B. ${ }^{2}$ \\ ALTARUGIO, Rafaela ${ }^{2}$ \\ ARBELLI, Rodrigo $\mathrm{O}^{2}$
}

\begin{abstract}
RESUMO: As deformidades angulares dos membros podem ocorrer devido à malformação óssea, dietas ricas em cálcio, consolidação inadequada de fraturas ou fechamento precoce das linhas epifisárias distais dos ossos longos. As epífises distal e proximal do rádio crescem em proporções diferentes das epífises ulnares, a interrupção do crescimento da ulna, devido ao fechamento precoce das linhas epifisárias, restringe o crescimento longitudinal do rádio, resultando no desenvolvimento de uma deformidade angular óssea denominada rádio curvo. O tratamento do rádio curvo é cirúrgico sendo que a técnica cirúrgica mais utilizada é a ostectomia ulnar associada a um enxerto de gordura autógena e fixador externo. Este trabalho tem como objetivo relatar a eficácia do tratamento para correção do rádio curvo em um cão, enfatizando o uso de um procedimento cirúrgico pouco convencional que consiste na técnica de ostectomia ulnar e radial em cunha associada à osteossíntese com placas e parafusos.
\end{abstract}

Palavras-chave: Rádio. Curvo. Ostectomia.

SUMMARY: The angular limb deformities may occur due to bone malformation, increasing levels of calcium in the diet, fracture with incomplete consolidation or premature closure of the distal physis of the long bones. The distal and proximal epiphysis of the radius grow at different rates of ulnar epiphyses, the ulnar interruption development, due to premature closure of epiphyseal lines, restricts the longitudinal growth of the radius, resulting in the development of an angular bone deformity called radius curvus. The treatment is surgical and consists in ulnar ostectomy associated with an autologous fat grafting and external fixator. This paper aims to report the effectiveness of treatment for radius curvus, emphasizing use of a surgical procedure which consists of the unconventional technique for radial and ulnar wedge ostectomy, associated with internal plates and screws fixation.

Keywords: Radius. Curvus. Ostectomy.

\section{INTRODUÇÃO}

As deformidades angulares consistem em desvios encontrados nos eixos dos membros anteriores e posteriores dos animais domésticos que podem ser causados por alterações congênitas ou adquiridas (AZEVEDO, 2002; BRINKER, PIERMATTEI, FLO, 2006).

Os desvios angulares são classificados como valgo ou varo quando ocorrem, lateralmente ou medialmente ao eixo ósseo central respectivamente (AZEVEDO, 2002; BRINKER, PIERMATTEI, FLO, 2006).

A etiologia das deformidades angulares dos membros ainda não é muito bem esclarecida, porém, considera-se que a forma congênita dessas afecções ocorra devido a alterações que culminam para a

\footnotetext{
${ }^{1}$ Docente - Dr. em Cirurgia Veterinária - UNIPINHAL/FAFRAM

${ }^{2}$ Acadêmicos do Curso de Medicina Veterinária do Centro Regional Universitário de Espírito Santo do Pinhal - SP (UNIPINHAL).
} 
malformação óssea, podendo estar relacionadas ao mau posicionamento intrauterino do feto, flacidez articular do feto em desenvolvimento, hipoplasia dos ossos carpianos e desequilíbrios hormonais. As formas adquiridas estão associadas a traumatismos, dietas ricas em cálcio que ocasionam alterações no desenvolvimento ósseo, exercícios excessivos, consolidação inadequada de fraturas ou devido ao fechamento precoce das linhas epifisárias distais dos ossos longos (HAZEWINKEL, 1989; BRINKER; PIERMATTEI; FLO, 2006; FOSSUM, 2008).

As epífises distal e proximal do rádio crescem em proporções diferentes das epífises ulnares, a interrupção do crescimento da ulna, em decorrência do fechamento precoce das linhas epifisárias, restringe o crescimento longitudinal do rádio. Como conseqüência, ocorre o encurtamento e o arqueamento cranial do rádio, o desvio lateral do carpo e a sub-luxação da articulação úmero-rádio-ulnar, resultando no desenvolvimento de uma deformidade angular óssea denominada rádio curvo (SILVA; RODIGHERI 2009).

O grau de deformidade angular e as discrepâncias de comprimento entre o rádio e ulna, podem ser determinados através de exames radiográficos (FOSSUM, 2008; HILTON, CHRISTOPHER, 2008).

Visando descrever uniformimente o grau, a direção e a relação das deformidades angulares dos ossos longos dos pequenos animais, foi criado um sistema de classificação denominado Center of Rotation of Angulation (CORA). A metodologia do CORA baseia na compreensão da relação existente entre os eixos anatômicos e mecânicos do osso anormalmente angulado e a articulacões adjacentes (PALEY, 2002).

As deformidades angulares dos membros podem ser corrigidas através da técnica de osteotomia com cunha de fechamento, ou através da técnica de ostectomia em cunha, com a finalidade de restaurar o alinhamento adequado das superfícies proximal e distal do membro afetado. A ostectomia em cunha preserva o comprimento ósseo, enquanto a osteotomia com cunha de fechamento proporciona uma superfície de fratura mais estável. Ambos procedimentos podem ser estabilizados com o uso de placas ósseas ou fixadores externos, que se tornaram as técnicas cirúrgicas de eleição (HAY; MANLEY, 1996; BRINKER; PIERMATTEI; FLO, 2006; FOSSUM, 2008; VANDEWATER; OLMSTEAD, STEVENSON, 2008).

O tratamento do rádio curvo é cirúrgico, visando que a destruição das células de crescimento ósseo e o fechamento da linha epifisária são irreversíveis e, portanto, o procedimento cirúrgico é direcionado a redução ou a correção das seqüelas ao fechamento epifisário prematuro (SILVA; RODIGHERI , 2009).

A técnica cirúrgica mais comumente utilizada para o tratamento do rádio curvo consiste na ostectomia ulnar, objetivando liberar a restrição de crescimento do rádio imposta pela ulna, associada a um enxerto de gordura autógena visando impedir a união dos segmentos ulnares, há também a necessidade de se fazer uso de fixadores externos que estabilizam a ostectomia e promovem a osteossíntese (SILVA; RODIGHERI, 2009).

Este trabalho tem como objetivo relatar a eficácia do tratamento para correção da deformidade 
angular do rádio (rádio curvo) em um cão, enfatizando o uso de uma técnica pouco convencional para correção desta afecção, que consiste na técnica de ostectomia ulnar e radial em cunha associada à osteossíntese com placas e parafusos.

\section{MATERIAIS E MÉTODOS}

Foi atendido no Hospital Veterinário (HOVET) do Centro Regional Universitário de Espírito Santo do Pinhal (UNIPINHAL), um cão SRD, macho, errante, com aproximadamente 2 anos de idade, pesando $25 \mathrm{~kg}$, apresentando deformidade angular em membro torácico direito. Em função do desvio angular o animal apresentava apoio leve com eventual impotência funcional do membro (Figura 1).

Figura 1. Imagem fotográfica evidenciando a presença de deformidade angular em membro torácico direito do animal (seta)

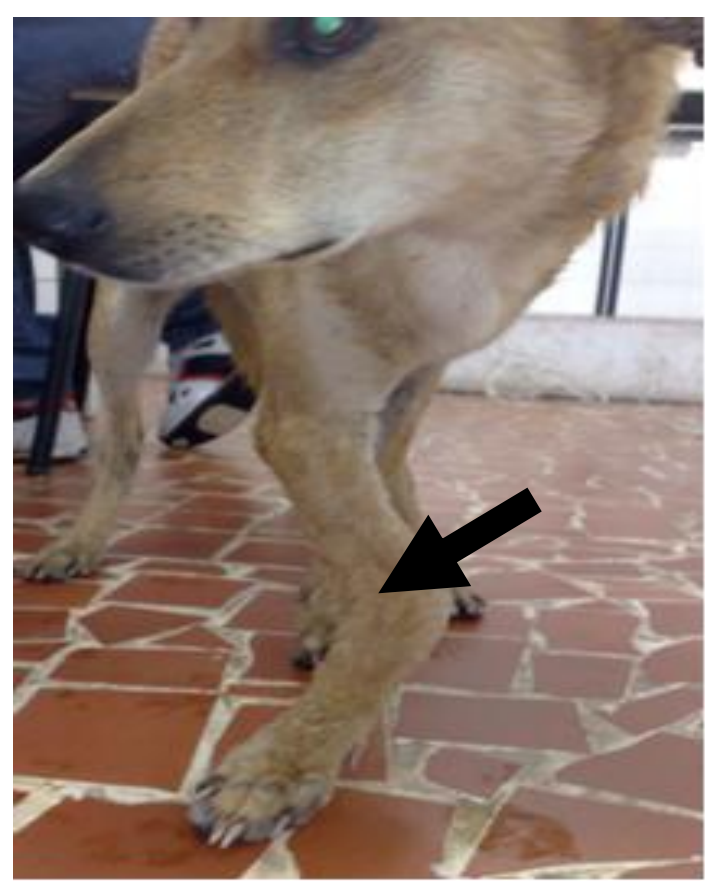

Fonte: Arquivo pessoal.

Após ser submetido à anamnese e exames físicos, o animal foi encaminhado ao setor de Diagnósticos por Imagem do HOVET, para realização de exames radiográficos, com o intuito de avaliar a gravidade da deformidade óssea.

À analise radiográfica observou-se a presença de um desvio crânio-medial em membro torácico direito denominado recurvatum, caracterizado pela angulação cranial do segmento distal do membro. Baseando-se nos sinais clínicos do paciente e nos achados radiográficos estabeleceu-se o diagnóstico da presença de desvio angular em varo no rádio direito do animal (Figura 2). 
Figura 2. Imagem radiográfica crânio-caudal evidenciando o rádio e a ulna direita: desvio angular crânio-medial: "rádio curvo".

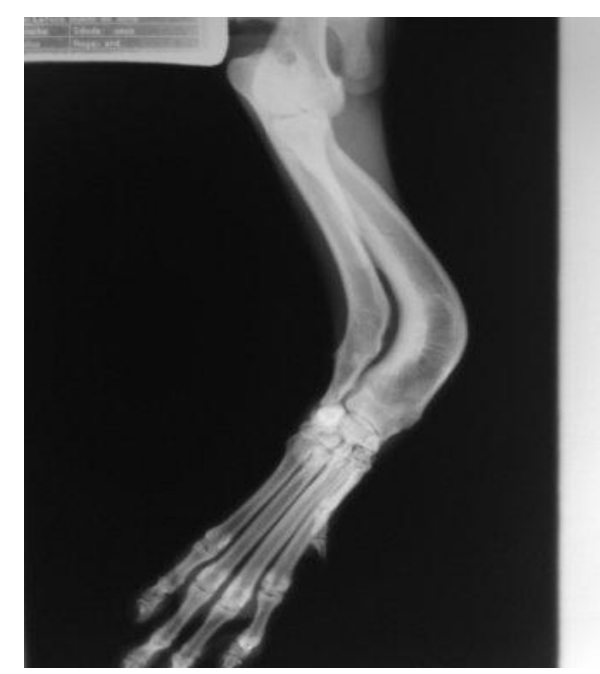

Fonte: Arquivo pessoal.

O exame radiográfico auxiliou na determinação exata do desalinhamento ósseo, serviu de ferramenta para identificação do CORA (Center of Rotation of Angulation) e avaliação dos pontos para ostectomia

Considerando-se os fatores etiológicos capazes de promover deformidades angulares nos membros, acredita-se que nesse caso, por se tratar de um animal errante, o mesmo possa ter sofrido algum trauma responsável por ocasionar uma fratura epifisária distal da ulna cuja consolidação errônea proporcionou a deformidade angular no membro afetado.

O tratamento de eleição preconizado para este caso foi realizado mediante a técnica cirúrgica da ostectomia ulnar e radial em cunha, associada à osteossíntese com placas e parafusos.

O protocolo anestésico adotado consistiu na administração de Acepromazina ${ }^{3}$ 0,2\% 0,05 mg/kg e Meperidina ${ }^{4} 4 \mathrm{mg} / \mathrm{kg}$ como medicação pré-anestésica. Para indução anestésica utilizou-se Propofol ${ }^{5} 5$ $\mathrm{mg} / \mathrm{kg}$, e para manutenção anestésica foi administrado Isoflurano ${ }^{6}$.

Para confecção da ostectomia corretiva, o animal foi posicionado em decúbito dorsal. Para $\quad$ o acesso cirúrgico do osso rádio foi realizada uma incisão crânio-lateral na pele, desde o terço médio do antebraço, estendendo-se até a região dos ossos do carpo, seguida da incisão do tecido subcutâneo, fáscia antebraquial e fáscia profunda a qual foi incisada ao longo da borda cranial do músculo extensor digital comum.

\footnotetext{
${ }^{3}$ Acepran ${ }^{\circledR} 0,2 \%$ - Univet, São Paulo - SP.

${ }^{4}$ Dolantina $^{\circledR} 50 \mathrm{mg} / \mathrm{ml}$ - Hoechst, São Paulo - SP.

${ }^{5}$ Propofol $^{\circledR} 10 \mathrm{mg} / \mathrm{ml}$ - Abbott, São Paulo - SP.

${ }^{6}$ Isoflurano ${ }^{\circledR} 100 \mathrm{ml}$ - Cristália, São Paulo - SP.

Nucleus Animalium, v.4, n.2, nov.2012
} 
A incisão do músculo abdutor longo do $1^{\circ}$ dedo foi feita na região localizada próxima a sua origem na ulna e paralela ao músculo extensor longo do $1^{\circ}$ dedo.

O afastamento dos músculos extensores foi realizado completando a exposição da diáfise radial e ulnar dos fragmentos fraturados, permitindo a identificação do ponto da ostectomia.

A ostectomia foi realizada com o auxílio de uma serra oscilante acoplada a uma furadeira de alta rotação, promovendo uma ostectomia ulnar cuneiforme.

Para osteossíntese foi utilizada uma placa DCP (Dynamic Compression Plate) neutra, de 3,5 mm; de 7 furos, 6 parafusos de $14 \mathrm{~mm}$ e 1 parafuso de $16 \mathrm{~mm}$ (Figura 3).

Figura 3. Osteossíntese: placa DCP neutra, $3,5 \mathrm{~mm}$; de 7 furos.

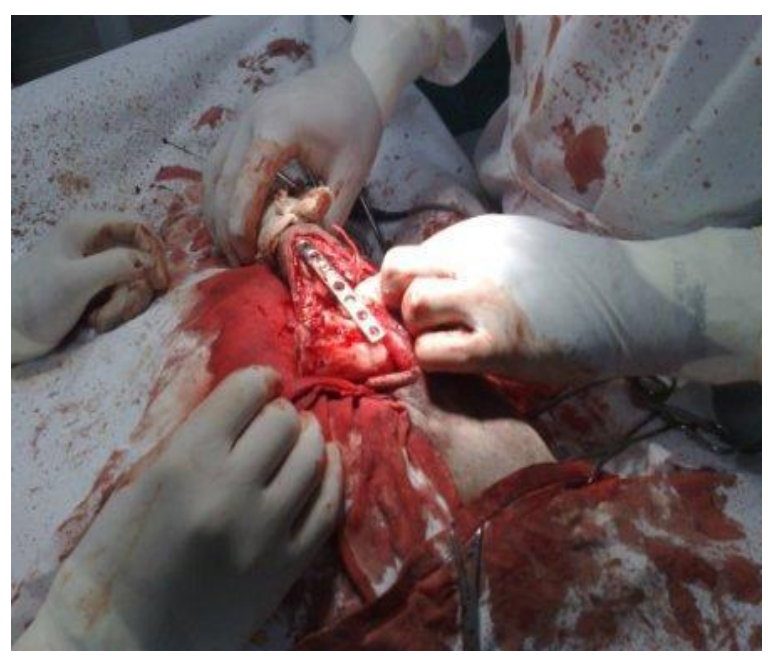

Fonte: Arquivo pessoal.

Realizou-se a síntese utilizando-se os fios de sutura de Poligalactina 0 no subcutâneo e PSS (Ponto Simples Separado) com Nylon 0 na pele.

O pós-operatório foi conduzido mediante administração do antibiótico Cefalexina ${ }^{7} 22 \mathrm{mg} / \mathrm{kg}$, BID; do antiinflamatório Carprofeno ${ }^{8} 4,4 \mathrm{mg} / \mathrm{kg}$, SID, durante 10 dias; dos analgésicos Dipirona Sódica ${ }^{9}$ $27,5 \mathrm{mg} / \mathrm{kg}$, TID, durante 10 dias e Tramadol ${ }^{10} 2,0 \mathrm{mg} / \mathrm{kg}$; TID, durante 10 dias; Rifampicina Spray ${ }^{11}$, aplicando-se 3 vezes ao dia sobre os pontos de sutura, durante 15 dias para o tratamento tópico e através do uso do Colar Elisabetano, visando impedir o contato do animal com o local abordado cirurgicamente.

Duas semanas após a cirurgia o animal já apresentava apoio efetivo do membro, não havendo a necessidade de realização de fisioterapia (Figura 4).

\footnotetext{
${ }^{7}$ Keflex ${ }^{\circledR} 50 \mathrm{mg} / \mathrm{ml}$ - Eurofarma, São Paulo - SP.

${ }^{8}$ Carproflan ${ }^{\circledR} 25 \mathrm{mg}$ - Pfizer Saúde Animal, São Paulo - SP.

${ }^{9}$ Novalgina ${ }^{\circledR} 50 \mathrm{mg} / \mathrm{ml}$ - Medley, São Paulo - SP.

${ }^{10}$ Tramal $^{\circledR} 50 \mathrm{mg} / \mathrm{ml}$ - Eurofarma, São Paulo - SP.

${ }^{11}$ Rifocina ${ }^{\circledR} 10 \mathrm{mg} / \mathrm{ml}$ - Eurofarma, São Paulo - SP.
} 
Figura 4. Apoio efetivo do membro torácico direito (seta).

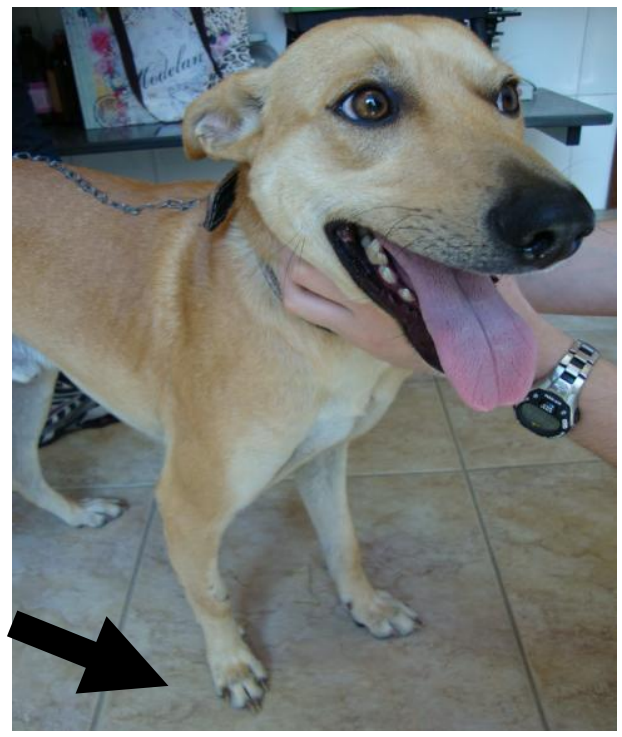

Fonte: Arquivo pessoa

Noventa dias após o procedimento cirúrgico foi observado o retorno do eixo ósseo e seis meses após o tratamento evidenciou-se o remodelamento ósseo do membro afetado.

\section{RESULTADOS E DISCUSSÃO}

A técnica cirúrgica mais comumente empregada no tratamento do rádio curvo consiste na ostectomia ulnar associada a enxerto autógeno de gordura e colocação de fixador externo para estabilização óssea (BRINKER, PIERMATTEI, FLO, 2006; SILVA, RODIGHERI 2009).

O prazo médio para o restabelecimento funcional do membro afetado é de 30 dias após o procedimento cirúrgico. O prognóstico para a aparência e a função normal do membro acometido depende do local da lesão e do tempo transcorrido após a oclusão epifisária (WOLTERS, 2004; SILVA, RODIGHERI 2009).

Observa-se no caso descrito que a técnica de ostectomia ulnar e radial em cunha associada à osteossíntese com placa e parafusos, utilizada para correção da deformidade angular apresentada pelo animal, consistiu em um procedimento cirúrgico efetivo no restabelecimento da função normal do membro, pois além de proporcionar um tempo de recuperação mais breve do que o descrito na literatura também eliminou os riscos de infecção e o desconforto pós-operatório promovidos pela presença do fixador externo.

\section{CONCLUSÃO}

Conclui-se que a ostectomia ulnar e radial em cunha, associada à osteossíntese com placa e parafusos, consiste em uma técnica cirúrgica eficaz apesar de ser pouco empregada. Os resultados esperados com o uso dessa técnica, de fato foram alcançados, pouco tempo após a cirurgia foi possível Nucleus Animalium, v.4, n.2, nov.2012 
observar o realinhamento do eixo ósseo, o restabelecimento da função normal do membro e o apoio precoce do membro.

Considerando-se a técnica cirúrgica mais usualmente aplicada para o tratamento da deformidade angular do rádio curvo, ressalta-se com o caso descrito neste trabalho que outros procedimentos cirúrgicos, tais como uso de placa e parafusos, visando à correção das deformidades angulares dos membros, podem ser empregados e aperfeiçoados, garantindo uma recuperação mais rápida e menos traumática, proporcionando um prognóstico favorável para o animal.

\section{REFERÊNCIAS}

AZEVEDO, J. R. de.et al. Deformidades angulares em bovinos: aspectos radiológicos e morfológicos, Maringá, PR, Brasil. In: ENCONTRO ANUAL DE INICIAÇÃO CIENTÍFICA, 11. Anais... Maringá, n. 6, p. 14-18, out. 2002.

BRINKER, W.O.; PIERMATTEI, D. L.; FLO, G. L. Handbook of small animal orthopedics and fracture. 4 ed. Philadelphia: Saunders, 2006. 807p.

FOSSUM, T. W. Cirurgia de pequenos animais. 3 ed. São Paulo: Elsevier, 2008. 1632p.

HAY, C. W.; MANLEY, P. Use of the Wagner apparatus to lengthen the antebrachium of a growing dog. Journal of Small Animal Practice, v. 37, n. 11, p. 543-548, nov. 1996.

HAZEWINKEL, H. A. W. Nutrition in relation to skeletal growth deformities. Journal of Small Animal Practice, v. 30, n. 11, p. 625-630, nov. 1989.

HILTON J.; CHRISTOPHER, J. Correction of angular limb deformity in a 4 month old German Shepherd. Cornell University Library, Ithaca - New York, jan. 2008.

PALEY, D. Principles of deformity correction. Berlin: Springer-Verlagg, 2002. 831p.

SILVA, N.S.; RODIGHERI, S.M. Correção de deformidade angular do rádio em um cão mediante a técnica de ostectomia ulnar. In: CONGRESSO CIENTÍFICO DA REGIÃO CENTRO-OCIDENTAL DO PARANÁ, Anais... Paraná n. 3, maio 2009.

VANDEWATER, A.; OLMSTEAD, M.; STEVENSON, S. Partial ulnar osteotomy with free autogenous fat grafting for treatment of radius curvus in the dog. Veterinary Surgery, v. 11, n. 3, p. 92-99, jul. 1992.

WOLTERS, S. A. B I.et al. Radius curvus syndrome in an african wild dog (Lycaon pictus). European Association of Zoo and Wildlife Veterinarians, Ebeltoft - Dinamarca, maio, 2004. 
